\title{
COMPUTATIONAL AND EXPERIMENTAL STUDY ON ION WIND SCHEME BASED AEROSOL SAMPLING FOR BIOMEDICAL APPLICATIONS
}

\author{
Tung Thanh Bui ${ }^{1}$, Thien Xuan Dinh ${ }^{2}$, Canh-Dung Tran ${ }^{3}$, Trinh Chu Duc ${ }^{1}$, and Van Thanh Dau \\ ${ }^{1}$ University of Engineering and Technology, Vietnam National University, Vietnam \\ ${ }^{2}$ Graduate School of Science and Engineering, Ritsumeikan University, Japan \\ ${ }^{3}$ School of Mechanical and Electrical Engineering, University of Southern Queensland, Australia \\ ${ }^{4}$ Research Group (Environmental Health), Sumitomo Chemical. Ltd, Hyogo, Japan
}

\begin{abstract}
In this paper, an efficient electrostatic particle sampling system is developed based on the neutralized ion wind. Compare with the conventional schemes where unipolar ion is used to charge the airborne sample and the sampling stage is fixed as a part of high voltage circuit, the new approach allows sampling stage to be electrically floated and adds insignificant charge to the bioaerosol, thus reduces damages to the microorganisms while provides design flexibility and good collecting efficiency. The approach is suitable for the combination with a microfluidic interface to develop complex aerosol-tohydrosol bio-samplers.
\end{abstract}

\section{KEYWORDS}

Aerosol sampling, particle charging, ionic wind, particle simulation, corona discharge, OpenFOAM

\section{INTRODUCTION}

Particle sampling in biotechnology, sampling the aerosol and transporting the particles to the detection unit, has recently attracted the researchers to develop new techniques in various application areas, especially in biomedical engineering. The objective of particle sampling is to take a representative sample of the ambient air and to transport it to the detection unit while keeping the aerosol concentration and size distribution unchanged. There are several active sampling devices including impactor, impinger, cyclone and filter, working based on the three

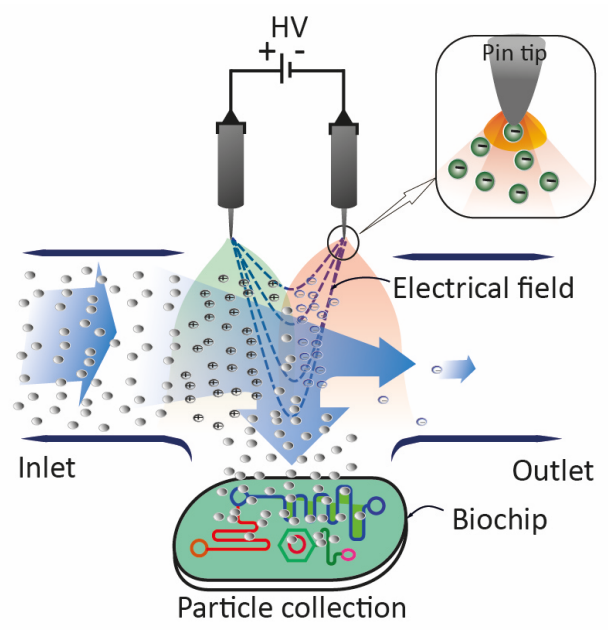

Figure 1: Configuration of the proposed bioaerosol electrostatic sampler. major collection methods: the impaction, impingement and filtration [1].

Among the particulate control devices, electrostatic precipitators (EPs) have been evaluated as an efficient technique to remove nanoparticles at a low pressure drop. Hence, the particle sampling based on the electrostatic particle precipitation is usually employed in bioengineering where biological samples are charged and exposed to an electric field. This yields a migration of particles deposited on a charged plate. This technique provides much softer collection for stress sensitive microorganism because the landing velocity on the substrate is usually much smaller than those by the impactor or impinger [2]. In this paper, we report the development of an efficient system based on ion wind scheme to monitor an aerosol-to-hydrosol sampler.

\section{WORKING PRINCIPLE}

In principle, a traditional Bio-aerosol Electrostatic Precipitators (ESP) is configured by one discharge electrode and one counter electrode in typical wire-tocylinder [3] where discharge electrode is to generate unipolar charge while the counter one is to define the electric field in parallel with or normal to the aerosol flow and also acted as the sampling electrode to collect particles. Since the conventional ESP creates considerably high charge, it is not suitable for bio-sampling. Indeed, it is difficult to segregate charged droplets from a plate due to the presence of Van-de-Wall force. Although this obstacle can be overcome using a solvent on the lab-on-chip interface named as the aerosol-to-hydrosol transfer [4][5] which attracts researchers for bio-aerosol sampling.

A mechanism of the proposed aerosol sampling approach is shown in Figure1, in which aerosol is directed to move in a desired trajectory toward the target by an ion wind in a channel without any net charge. The principle has been presented in environmental sensing [6], [7] and is applied in the present scheme as follows. An electric field caused by a high voltage isolated from the sampling collector is applied between two discharge electrodes to generate ionized wind. By the effect of the electric field and bipolar ionized wind, particles are neutralized and gently shifted toward the collection cavity of the channel [8]-[10]. Since neither electrode nor sampling chip is installed inside the channel, there is not any obstacle through the channel. Hence, the kinetics of particle flow and ion wind can be efficiently handled by experiments and easily simulated. As the result, airborne particles are directed to move along 
desire trajectories by a controllable ion wind to reach the collection cavity without any net charge.

Due to the isolation of sampling chip from the ion wind generating circuit, its material can be chosen arbitrarily for the designing request such as glass, gel, metal or semiconductor. This is one of the strong points of the method. Indeed, in previous publications of this approach [11], since the chip is a component of high voltage circuit, accumulated particles on the chip can ignite the discharge from the chip and then ultimately damage bio-particles. Another advantage of the present work is to keep particles on the chip owing to the electrostatic force for several purposes and analyses.

\section{NUMERICAL SIMULATION}

A numerical modelling is carried out using OpenFOAM finite volume based solver to simulate the

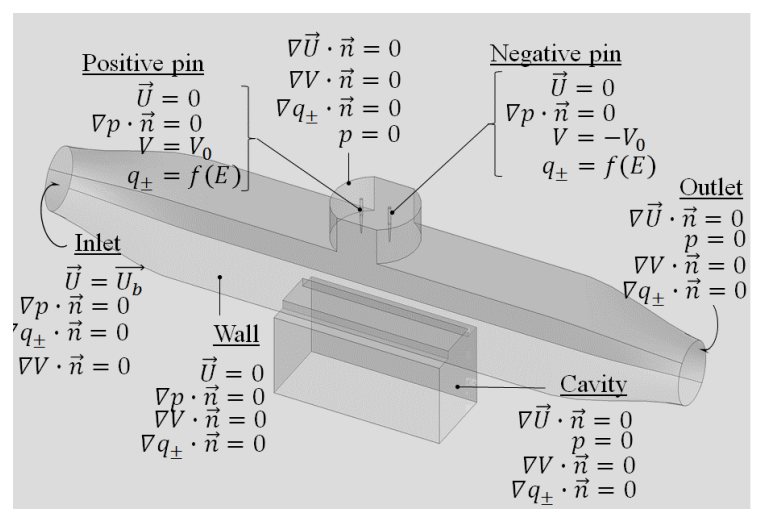

Figure 2: Simulation of aerosol sampler: consideration domain and boundary conditions. For the electric field, a given voltage is applied on the boundary of electrodes and a given Neumann condition is applied at the edges of the considered domain. At the electrode, we assume that the corona discharge has got a constant ion density $\rho_{ \pm}=I /\left(\mu E_{w} A\right)$ where $A$ is the total area of the tip.

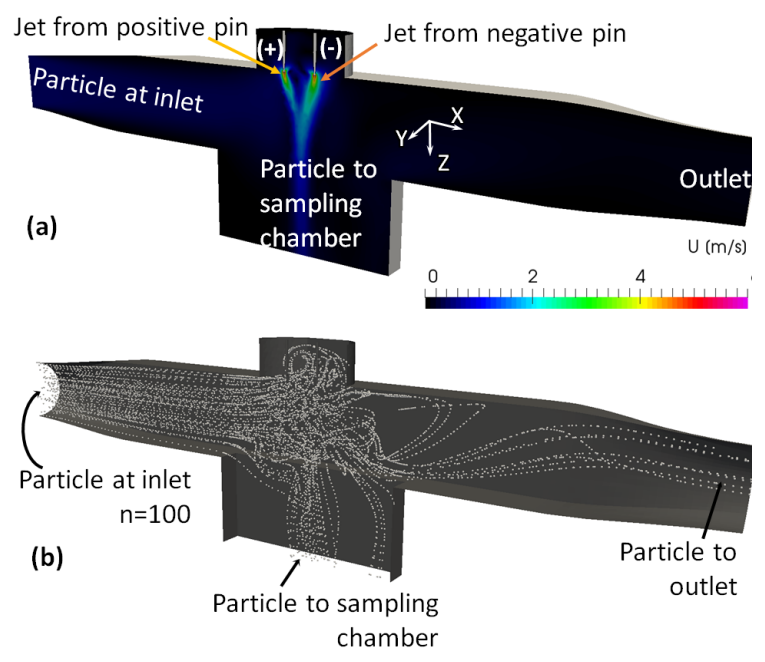

Figure 3: Numerical simulation of the present sampler by FEM using Open-FOAM. (a) Geometry of the problem and (b) Trajectories of particles in channel. flow of particles under the effect of an ion wind [12]. A 3$\mathrm{D}$ domain under consideration is described in Figure 2 together with boundary conditions verified by experiments on both electrical field and CFD to simulate the ion wind caused by a corona discharge process. At the inlet, an input flow of one-hundred particles whose diameter is $1 \mu \mathrm{m}$ moves in the channel with a uniform velocity $U_{b}$. The efficiency of the proposed sampling method is based on the numbers of input particles, output particles going through the channel to the outlet, and the number of collected particles at the collection cavity.

Figure 3(a) represents the simulated ionic wind by electrodes. Particles' trajectories in the aerosol sampler with the corona whose parameters are described above is shown in Figure 3b. The numerical solution which is carried out with a flowrate of $5 \mathrm{lpm}$ indicates that $65 \%$ of number of particles approaching to the collector with corona by an applied voltage of $4.0 \mathrm{kV}$ versus $20 \%$ without corona using the mentioned parameters.

\section{EXPERIMENT SETUP}

A prototype of the sampler has been developed as shown in Figure 4. The aerosol produced by a nebulizer is particulate suspension consisting of $1 \mu \mathrm{m}$ polystyrene particles and other mixing ones, for example water particles and measured by a portable aerosol meter.

The corona wind generator is installed on one side and in the middle of the channel while the cavity chamber collecting particles is placed on the opposing side and linked with the channel via a slit of $8 \mathrm{~mm} \times 30 \mathrm{~mm}$. The ion wind generator consists of two stainless steel SUS304 electrodes of $8 \mathrm{~mm}$ long each and $0.4 \mathrm{~mm}$ in diameter which are disposed in parallel with each other. These pin electrodes with tip size of $80 \mu \mathrm{m}$ are fixed inside of a pin holder applied by an insulated coating to ensure the electrical isolation of the high voltage from surroundings. The tip of the discharge electrodes is covered up $2 \mathrm{~mm}$ away from the wall by an adjustable probe installed in the channel to limit the effect of pin shape on the dynamics of flows of particles and ion wind as well as to protect the flows from the electrodes' contaminant.

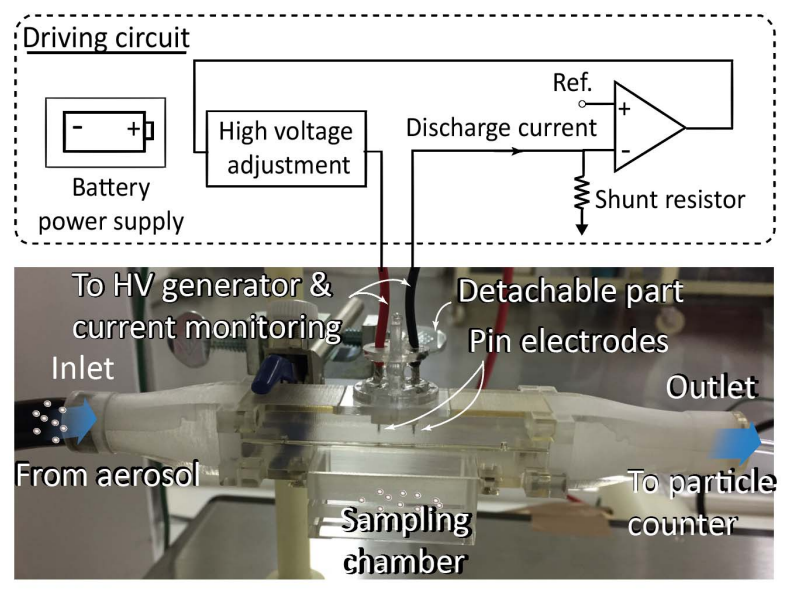

Figure 4: The present bio-aerosol electrostatic sampler (BES): Schema of the BES together with its configuration and principle. 
In order to generate ion wind, a high voltage is applied between electrodes and the discharge current is measured at the negative electrode. In order to prepare the experiment, the air velocity is determined using a conventional air flow meter ISA79 with 10-mm hotwire and range of $10 \mathrm{~ms}^{-1}$ to establish the velocity profile inside the channel through 3-dimensional computational fluidic dynamics simulation. Furthermore, the temperature and the net charges of air flow fed to the channel are also recorded before the experiment. Through the experiment, the current and voltage $(\mathrm{I}-\mathrm{V})$ characteristic of the corona is recorded for a range of air flow rates. The time-resolved current is also recorded using a HAMEG-R\&S oscilloscope with a range of air flow velocity to investigate the repeatability and hysteresis of the measured current.

The performance and efficiency of the present bioaerosol sampling method are investigated based on the numbers of input particles from nebulizer at the entrance, the particles going through to the downstream and the total ones reaching the cavity chamber of the channel. This measurement is carried out with an initial number of particles from nebulizer using a portable aerosol meter through the experiment for a range of different flow velocities with various corona discharge voltages to model efficiently a real bio-aerosol sampling.

In this work, a dilute particulate suspension of dyed polystyrene particles with diameter of approximately $1 \mu \mathrm{m}$ (Thermo-Fisher Scientific) was used. The sample of particles is stored in room condition for 30 minutes after removing water by a filter of $0.2 \mu \mathrm{m}$ nano-porosity (Merck Millipore Ltd). The particles are then stirred up by a mild ultrasonic mixer to prevent the particles' agglomeration before being introduced into a nebulizer. Although the nebulizer can operate with a wide range of particle size as natural breathes, the trace by particle of $1.0 \mu \mathrm{m}$ helps investigate the performance of device more reliable. The measurement protocol is carried out as follows. One minute after turning on the nebulizer which is connected to the inlet of the channel, the voltage is applied to induce corona discharge current. Until a stable current is achieved, the aerosol meter is switched on to start measuring the particle concentration every 20 seconds. This measurement process is repeated five times. In order to characterise the new approach based aerosol sampler, the experiment is conducted with a range of flow rates of the nebulizer which is controlled by a compressor and measured at the channel's output using AWM5102VN flow sensor (Honeywell, USA). All obtained data on the particle counter together with the corresponding flow parameters will be recorded to express the feature of the bio-aerosol sampler.

\section{RESULTS AND DISCUSSION}

In this work, the flow rate of particles is set up from 1 to to $6 \mathrm{lpm}$ while determine the corona current in order to characterise the aerosol sampler. Since the maximum velocity of aerosol (particle flow) is $0.53 \mathrm{~m} / \mathrm{s}$ (under 1.0 $\mathrm{m} / \mathrm{s}$ ) corresponding to the flow rate of $6 \mathrm{lpm}$, it does not affect the corona discharge [13]. Furthermore, the particles' diameters used in the experiment is $1 \mu \mathrm{m}$. The collection efficiency of the sampler for a flow rate and applied voltage is defined as follows.

$$
\eta=1-\frac{N_{\text {outlet-On }}}{N_{\text {outlet-off }}}
$$

where $\mathrm{N}_{\text {outlet-on }}$ and $\mathrm{N}_{\text {outlet-off }}$ are the aerosol particle concentrations at the channel outlet when corona discharge is on and off, respectively and measured by the aerodynamic particle sampler (APS). For several published cases the collection efficiency in a unipolar charge system is predicted by Deutsch-Anderson equation

$$
\eta=1-\mathrm{e}^{-\frac{n_{p} e C_{C} E}{3 \pi \mu d p} \times \frac{A}{Q}}
$$

where $\mathrm{A}$ is the collecting area; $n_{p}$ the particle charge; e the elementary charge; $C_{C}$ the slip correction factor; $\mathrm{E}$ the electric field; $\mu$ the flow viscosity; $Q$ the flow rate of aerosol and $d_{p}$ the particle diameter.

The equation Eq. (2) depicts that collection efficiency increases with the increasing of particle charge $\left(n_{p}\right)$ and/or the decreasing of flow rate (Q). Furthermore, since the particle charge increases with the ion wind concentration and then with the increasing of voltage, the collection efficiency increases with the increase of voltage.

We could confirm the sampling performance of the proposed aerosol sampler. Figure 5 illustrates the images of particle collected at the sampling chamber when high voltage on and off. The particle density measured at the collection cavity and the outlet with and without the

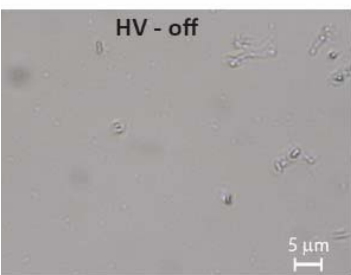

(a)

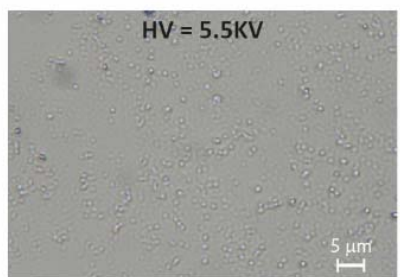

(b)
Figure 5: Experimental result showing images of particle collected at the sampling chamber when high voltage on and off.

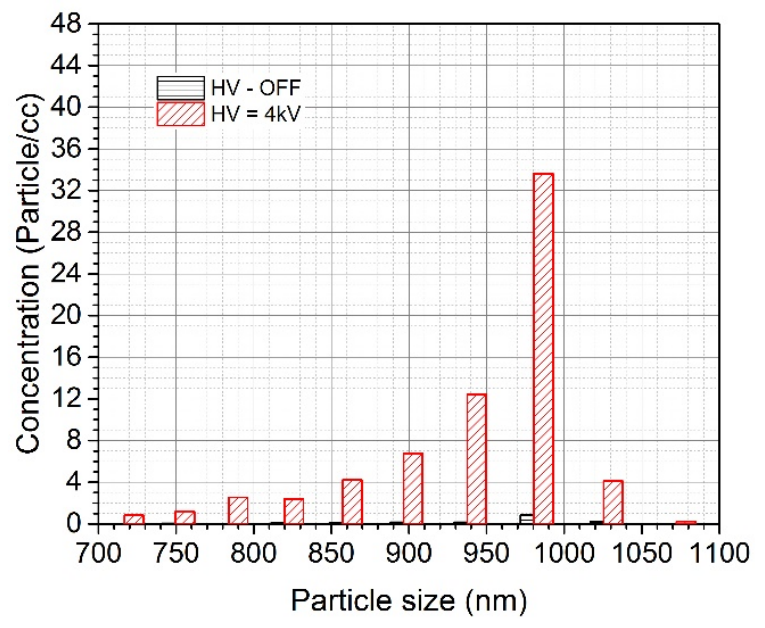

Figure 6: The collected particles by the experiment at the collection area. 


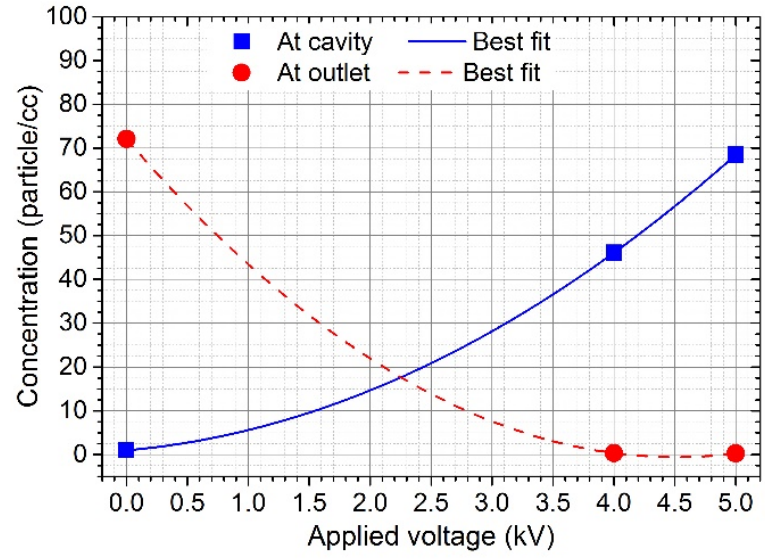

Figure 7: The efficiency of the particle collection by the experimnet.

presence of the ion wind is plotted in Figure 6. The experiment shown that with the presence of ion wind (it means a voltage is applied), almost particles move to the cavity to be collected there. Indeed, without the ion wind the density of particle flow measured at the outlet is almost the same with one measured at the collection cavity with the presence of the ion wind. It shows that particles were almost directed from the entrance of the channel to the cavity where they are collected as requested.

For our present aerosol sampler, the efficiency of the particle collection by Eq. 1 is determined from the experiment with a range of the aerosol flow rates (from 20 $1 \mathrm{pm}$ to $100 \mathrm{lpm}$ ) and the applied voltages of $5.5 \mathrm{kV}$ using particles of around $1.0 \mu \mathrm{m}$ diameter. Figure 6 describes the efficiency decrease with the increase of the aerosol flow rate at a constant applied voltage ( $4 \mathrm{kV}$ in this experiment). Indeed, the result shows that the efficiency reaches the maximum value of $95 \%$ and starts linearly decreasing from the flow rate of $4.2 \mathrm{lpm}$.

\section{CONCLUSION}

An aerosol sampling method has been developed based on the combination between a microfluidic interface and the ion wind scheme to monitor an aerosol-to-hydrosol sampler without any net charge. By the effect of the electric field and ionized wind, particles are neutralized and gently shifted toward the sampler. Since neither electrode nor sampling chip is installed inside the channel, flows of particle and ion wind are efficiently handled without any obstacle and the kinetics of the flows can be easily simulated in the channel. One of the advantages of the method is that the material of sampling chip can be chosen arbitrarily for the designing request such as glass, gel, metal or semiconductor owing to the isolation of sampling chip from the ion wind generating circuit. Furthermore, the present approach keeps almost particles on the chip owing to the electrostatic force as investigated by both experimental and simulation works. Although further investigations are necessary, a combination between ion wind based automated electrostatic sampler and a biosensor device developed by this work would yield a promising platform for a system of automated bio-aerosol sensing.

\section{ACKNOWLEDGMENT}

Tung Thanh Bui would like to acknowledge the Vietnam National Foundation for Science and Technology Development (NAFOSTED) for financial support through a project entitled "Sensing microfluidic living cell manipulation system based on dielectrophoresis technique", Grand Number "107.99-2016.36".

\section{REFERENCE}

[1] J. Mandal and H. Brandl, "Bioaerosols in Indoor Environment - A Review with Special Reference to Residential and Occupational Locations," Open Environ. Biol. Monit. J., vol. 41, no. 1, pp. 83-96, 2011.

[2] G. Mainelis, "Collection of Airborne Microorganisms by Electrostatic Precipitation," Aerosol Sci. Technol., vol. 30, no. 2, pp. 127-144, Feb. 1999.

[3] J.-M. Roux, O. Kaspari, R. Heinrich, N. Hanschmann, and R. Grunow, "Investigation of a New Electrostatic Sampler for Concentrating Biological and NonBiological Aerosol Particles," Aerosol Sci. Technol., vol. 47, no. 5, pp. 463-471, 2013.

[4] H. N. Phan and A. R. McFarland, "Aerosol-toHydrosol Transfer Stages for Use in Bioaerosol Sampling," Aerosol Sci. Technol., vol. 38, no. 4, pp. 300-310, 2004.

[5] M. Wubulihairen, S. Y. Jiang, and Z. Ning, "Prototype Development and Laboratory Evaluation of an Aerosol to Hydrosol Sampler," Aerosol Air Qual. Res., vol. 15, pp. 776-785, 2015.

[6] V. T. Dau, T. T. Bui, T. X. Dinh, and T. Terebessy, "Pressure sensor based on bipolar discharge corona configuration," Sensors Actuators A Phys., vol. 237, pp. 81-90, Jan. 2016.

[7] V. T. Dau, T. X. Dinh, T. T. Bui, and T. Terebessy, "Corona anemometry using dual pin probe," Sensors Actuators A Phys., vol. 257, pp. 185-193, 2017.

[8] V. T. Dau, T. X. Dinh, T. T. Bui, C. D. Tran, H. T. Phan, and T. Terebessy, "Corona based air-flow using parallel discharge electrodes," Exp. Therm. Fluid Sci., vol. 79, pp. 52-56, 2016.

[9] V. T. Dau, T. X. Dinh, T. Terebessy, and T. T. Bui, "Ion Wind Generator Utilizing Bipolar Discharge in Parallel Pin Geometry," IEEE Trans. Plasma Sci., pp. 1-9, 2016.

[10] V. T. Dau, T. X. Dinh, T. Terebessy, and T. T. Bui, "Bipolar corona discharge based air flow generation with low net charge," Sensors Actuators A Phys., vol. 244, pp. 146-155, 2016.

[11] G. Pardon, L. Ladhani, N. Sandström, M. Ettori, and G. Lobov, "Aerosol sampling using an electrostatic precipitator integrated with a microfluidic interface," Sensors Actuators B. Chem., vol. 212, pp. 344-352, 2015.

[12] V. T. Dau, T. X. Dinh, T. T. Bui, and T. Terebessy, "Bipolar corona assisted jet flow for fluidic application," Flow Meas. Instrum., vol. 50, pp. 252260, 2016.

[13] M. D. Yamanaka, H. Hirosawa, Y. Matsuzaka, and H. Tanaka, "Glow-discharge' ionic anemometer," Rev. Sci. Instrum., vol. 56, no. 4, pp. 617-622, 1985. 